\title{
Relations between Parenting and Adolescents' Academic Functioning: The Mediating Role of Achievement Goal Orientations
}

\author{
Xinpei Xu' ${ }^{1}$, David Dai ${ }^{2}$, Ming Liu $^{3}$ and Ciping Deng ${ }^{1 *}$ \\ 'Shanghai Key Laboratory of Brain Functional Genomics, School of Psychology and Cognitive Science, East China \\ Normal University, Shanghai, China, ${ }^{2}$ Department of Educational and Counseling Psychology, University at Albany \\ (SUNY), Albany, NY, United States, ${ }^{3}$ Department of Special Education, Faculty of Education, East China Normal University, \\ Shanghai, China
}

\section{OPEN ACCESS}

Edited by:

Jesus de la Fuente,

University of Almería, Spain

Reviewed by: Jesús Nicasio García Sánchez,

Universidad de León, Spain Martina Smorti,

University of Pisa, Italy

*Correspondence: Ciping Deng

cpdeng@psy.ecnu.edu.cn

Specialty section: This article was submitted to Educational Psychology, a section of the journal

Frontiers in Education

Received: 10 November 2017 Accepted: 05 January 2018

Published: 19 January 2018

Citation:

Xu X, Dai D, Liu M and Deng C (2018) Relations between Parenting and Adolescents' Academic Functioning: The Mediating Role of Achievement Goal Orientations.

Front. Educ. 3:1.

doi: 10.3389/feduc.2018.00001
We investigated whether and how adolescents' perceived parental psychological control and autonomy support influence their maladaptive academic functioning through their achievement goal orientations. Participants were 845 tenth-grade students (447 boys, $M_{\text {age }}=15.20 \pm 0.54$ years; 398 girls, $M_{\text {age }}=15.13 \pm 0.47$ years) in China. Data were collected on their reported achievement goal orientations, perceived parental psychological control and autonomy support, and academic-related beliefs, strategies, and behaviors. Bootstrapping with resampling strategies was used for testing multiple mediators' model and examining mediation effect. Results indicated that, compared with girls, adolescent boys perceived higher parental psychological control. Moreover, we found distinct effects of parental psychological control and autonomy support on adolescents' maladaptive academic functioning through achievement goal orientations. Specifically, parental psychological control led to adolescents' maladaptive academic functioning, mainly through adolescents' performance-approach goal orientation (PAP) $(0.06, \mathrm{BC} 95 \% \mathrm{Cl}[0.03,0.09])$ and performance-avoidance goal orientation (PAV) $(0.02, \mathrm{BC} 95 \% \mathrm{Cl}[0.01,0.03])$ rather than mastery goal orientation (MAS) $(-0.00, \mathrm{BC} 95 \% \mathrm{Cl}[-0.01,0.01])$; while parental autonomy support reduced adolescents' maladaptive academic functioning, mainly through their MAS $(-0.02, \mathrm{BC}$ $95 \% \mathrm{Cl}[0.11,0.19])$ rather than PAP $(0.01, \mathrm{BC} 95 \% \mathrm{Cl}[-0.01,0.03])$ and PAV $(0.01$, $\mathrm{BC} 95 \% \mathrm{Cl}[-0.01,0.02])$. The results suggest that adolescents will benefit from parents ameliorating maladaptive academic functioning through fostering MASs and be harmed from parents facilitating maladaptive academic functioning through enhancing performance-approach and PAVs.

Keywords: psychological control, autonomy support, achievement goal orientation, academic functioning, mediating effects

\section{INTRODUCTION}

Among the many constructs of parenting, psychological control and autonomy support have been the primary focus of many studies. Psychological control and autonomy support first appeared in the Children's Report of Parental Behavior Inventory (Schaefer, 1965). Initially, many scholars equated psychological control and the absence of autonomy support, computing one variable by 
reversing the other (Gray and Steinberg, 1999). Until later, Silk et al. (2003) asserted psychological control and autonomy support should be regarded as distinct parenting constructs rather than two ends of a continuum by revealing discrete factors for psychological control and autonomy support.

Psychological control refers to the display of a conditionally approving attitude toward children and involves engagement in a host of intrusive parenting tactics to make children think, behave, or feel in parentally approved ways (Barber and Harmon, 2002). Autonomy support is defined as the extent to which parents consider children's perspective, acknowledge children's feelings, encourage them to solve problems by themselves, and provide opportunities for choice (Deci and Ryan, 1985). A bulk of evidence has indicated that parental psychological control is detrimental, while parental autonomy support is beneficial for children's academic motivation and learning (e.g., Tynkkynen et al., 2012; Vasquez et al., 2016). However, most existing research focused on this issue has been conducted in Western settings, with a dearth on its relations among Chinese societies. Hence, this study investigated the relations between parental psychological control, autonomy support, and academic functioning in a sample of Chinese adolescents.

\section{Parental Psychological Control, Autonomy Support, and Academic Functioning: Research in the West}

The nature and effects of parental psychological control and autonomy support on children have been the focus of considerable research. In conceptualizing the roles of psychological control vs. autonomy support, researchers based on self-determination theory (Deci and Ryan, 2010) emphasize the importance of satisfying children's psychological autonomy for people's psychological adjustment and growth. Psychological autonomy is the experiences of inner volition and choice (Vansteenkiste et al., 2005). The main difference between parental psychological control and autonomy support is how parents treat children's autonomy. It is harmful to children's learning processes if children are coerced by externally or internally pressuring demands (Ryan and Deci, 2003). Therefore, many Western researchers assert that parental autonomy support is beneficial while parental psychological control is detrimental for children's academics.

In recent years, the differentiation the dimensions of psychological control and autonomy support has proved to enhance discriminant validity in terms of their effects on children's academic behavior. Empirically, children who receive autonomysupportive parenting show adaptive learning, such as positive attitudes toward school (Annear and Yates, 2010), high homework quality (Hagger et al., 2015), and academic performance (Vasquez et al., 2016; Froiland and Worrell, 2017). In contrast, children with psychologically controlling parents were likely to show maladaptive behaviors, such as intervened autonomy efforts (Vansteenkiste et al., 2005), negative attitudes toward learning (Gonzalez et al., 2002), and undesirable school achievement (Aunola and Nurmi, 2004).

\section{Parental Psychological Control, Autonomy Support, and Academic Functioning: Research in China}

Researchers argued that the relations between parental psychological control, autonomy support, and academic functioning may show differences in Asian cultures in which relatedness and interdependence than autonomy are more highly valued (Chao and Tseng, 2002; Nelson et al., 2006). Compared with Western counterparts, Chinese parents use more psychological controlling and less autonomy-supportive practices (Ng et al., 2014; Doan et al., 2017). In addition, Chinese parents and students might interpret parental psychological control differently from Western counterparts, treating it as the expression of love and discipline while not an intrusion upon children's sense of self (Wang et al., 2007; Soenens et al., 2012). Therefore, more use of parental psychological control and less autonomy support might be perceived as normal or appropriate in China, implies the harms of psychological control and the benefits of autonomy support to Chinese children's development may be weaker or even not appear.

A few studies have examined the effect of parental psychological control and autonomy support on children and adolescents' academic learning in China. For parental autonomy support, findings were consistent. Results indicated that autonomy support contributed to students' academic functioning. For instance, Vansteenkiste et al. (2005) found that parental autonomy support vs. psychological control was associated with more optimal learning attitudes and academic success. Moreover, Wang et al. (2007) found that autonomy support predicted adolescents' increased academic functioning over time, and these beneficial effects were weaker in China than in the United States.

As for psychological control, findings were inconsistent. Some studies found that psychological control had detrimental effects in adolescents' academics. For example, Wang et al. (2012) found that Chinese adolescents perceived higher parental psychological control had more poor school performance. In line with this, significant negative association between parental psychological control and academic performance was found among students in their late adolescence (Lu et al., 2017). However, there were also some studies demonstrating that psychological control had no significant relations with Chinese adolescents' academic functioning (Lin, 2001; Wang et al., 2007). Lee et al. (2012) even maintained that parental higher psychological control tended to produce greater achievement on condition children do not view it in a negative manner. Thus, due to these inconsistent findings, many researchers (e.g., Lu et al., 2017) asserted the necessity of further research to examine the relations between parental psychological control and adolescents' academic functioning within and across different countries and cultural context.

\section{The Mediating Role of Achievement Goal Orientations in the Relations between Parenting and Adolescents' Academic Functioning}

In addition to parental psychological control and autonomy support, personal achievement goal orientations also shape 
students' achievement strivings and influence their academic functioning (Grouzet et al., 2004). Achievement goal orientations refer to cognitive representations of the reason students engage in a task (Deci et al., 1996; Wolters, 2004). Achievement goal orientations originated in the late 1970s and early 1980 s with the work of Dweck and others (Ames, 1984; Nicholls, 1984; Dweck, 1986). Now widely used is a trichotomous achievement goal framework that includes mastery goal orientation (MAS), performance-approach goal orientation (PAP), and performance-avoidance goal orientation (PAV). MAS refers to the pursuit of improving one's ability and skills. Performanceapproach goal orientation focuses on proving one's competence and outperforming others. Performance-avoidance goal orientation refers to trying to avoid failure or looking incompetent.

The specific reasons for examining achievement goal orientations as mediators for the relations between parental psychological control, autonomy support and students' academic functioning can be summarized in two ways. First, there is substantial evidence on the influence of students' achievement goal orientations on their learning (Diseth and Samdal, 2014). Mastery-oriented students tend to use effective learning strategies, prefer challenging tasks, and have good grades (Diseth and Kobbeltvedt, 2010). Performance-avoidance goal orientation is positively associated with self-handicapping strategies and negatively with achievement (Diseth and Kobbeltvedt, 2010). In contrast, the relations between PAP and students' academic functioning are complex. Some studies found it was linked to positive learning outcomes, such as good task absorption, persistence, and high grades (Pastor et al., 2007). while other studies found students with PAP have test anxiety and shallow learning processes (Elliot et al., 1999). Second, the mediating possibility of achievement goal orientations on the relation between parental psychological control, autonomy support, and academic functioning is plausible based on the empirical evidence. Although, to our knowledge, no study has directly investigated the mediating role of achievement goal orientations between psychological control, autonomy support and academic functioning, the mediating role of achievement goal orientations has been identified when the relations between some parenting constructs and academic functioning are concerned. For instance, De Bruyn et al. (2003) found the mediating role of students' goal orientations on the relations between parental induction and students' academic success. Grolnick et al. (1991) and D'Ailly (2003) found that maternal autonomy-supportive parenting predicted children's motivational variables (e.g., perceived autonomy, control, and competence), and, in turn, these motivational variables positively predicted children's academic performance. Moreover, parental control predicted low academic achievement partially through students' high mastery-avoidance and PAVs (Luo et al., 2013). Despite the limited number, these extant studies imply that students' achievement goal orientations may play mediating roles in the relations between two types of parenting (psychological control and autonomy support) and students' academic functioning.

\section{The Present Study}

The primary goal of the present study was to extend the literature on the understanding of the relations between parenting and students' academic functioning in a Chinese context in two important ways. One is to examine the relations between parental psychological control, autonomy support, and adolescents' academic functioning in China; the other is to examine how achievement goal orientations are constructed and developed by psychological control and autonomy support and how they subsequently influence students' maladaptive academic functioning. We focused on students in adolescence given that during these phase parenting is particularly salient when teenagers are becoming more independent and individuated from their family (Eccles et al., 1993) and academics often get a heightened emphasis (for a review, see Pomerantz et al., 2008).

Based on the past research, we hypothesized that parental autonomy support would be positively and psychological control would be negatively linked to adolescents' academic functioning. However, due to the inconsistent findings in previous studies, we were also open to an alternative hypothesis that parental psychological control would not have a negative relation with adolescents' academic functioning. In addition, we also hypothesized that students' three types of achievement goal orientations would have mediating roles in the relations between parental psychological control, autonomy support and academic functioning.

\section{MATERIALS AND METHODS}

\section{Participants}

The sample consisted of 845 tenth-grade students (447 boys, $\mathrm{M}_{\text {age }}=15.20 \pm 0.54$ years; 398 girls, $\mathrm{M}_{\text {age }}=15.13 \pm 0.47$ years) in four selective high schools that were in five cities in China (Beijing, Guangzhou, Xian, Fuzhou, and Shenyang). Almost all adolescents in this sample were of the Han ethnicity. $98 \%$ of the adolescents were from intact families, and the others were living with one parent because of parental divorce, death, or other reasons. Most of the parents were at the age of 41-45 years old. In collected effective data, $93 \%$ of their fathers and $93.1 \%$ of the mothers had an educational level of high school or above; $93.5 \%$ of the fathers and $83.4 \%$ of the mothers had their full-time jobs.

\section{Procedure}

Students completed self-report measures of personal achievement goal orientations, academic-related functioning, and their perceived parental psychological control and autonomy support. The data were collected online in their first semester of the tenth grade. Every student had an account and password to log into online assessment system, which guaranteed participants' privacy and security and ensured the accuracy of results and automation of data collection. A certain psychology teacher with necessary training in each school organized the participants to complete the online measures in their computer rooms during the students' leisure time. Extensive explanations were provided to the participants if they had questions during the collection of data. No evidence indicated that the students had difficulties in understanding the measure items. Written consent was obtained from all students and their parents/legal guardians through the schools. 


\section{Measures}

The measures were initially created in English. Standard translation and back-translation procedures (Brislin, 1980) were followed to generate the Chinese versions. A variety of strategies (e.g., repeated discussion in the research group to modify the wording of the items, interviews with adolescents) were made to ensure that the Chinese versions keep similar meanings with the English versions (Erkut, 2010). In addition, linguistic factors were also taken into consideration so that the measures were understandable to adolescents in China.

\section{Psychological Control}

Adolescents reported on their parents' psychological control by responding to an 18-item measure. The items, most of which were selected from existing measures (Barber, 1996; Silk et al., 2003), were used to assess psychological control by Wang et al. (2007). Adolescents indicated how true each item was of their parents $(1=$ not at all true, $5=$ very true $)$. These items tapped authority assertion (e.g., "My Parents tell me that I should feel guilty when I do not meet their expectations."), guilt induction (e.g., "My parents act cold and unfriendly if I do something they do not like."), and love withdrawal (e.g., "My parents tell me that what they want me to do is the best for me and I should not question it."). The mean of all items was taken, with higher numbers indicating greater psychological control. The measure has proved valid and appropriate among Chinese adolescents (e.g., Ng et al., 2014). The confirmatory factor analysis indicated that construct validity of the instrument was acceptable in the present study, $\chi^{2}=622.81, d f=132, p<0.001$, RMSEA $=0.08$, $\mathrm{CFI}=0.89$, TLI $=0.88$. Internal reliability was high $(\alpha=0.94)$ in this study.

\section{Psychological Autonomy Support}

Ten items adopted from prior research were used to measure parental psychological autonomy support (McPartland and Epstein, 1977; Steinberg et al., 1992). Children indicated ( $1=$ not at all true, $5=$ very true) the extent to which their parents used autonomy-supportive practices (e.g., "My parents allow me to make choices whenever possible" and "My parents are usually willing to consider things from my point of view"). The mean of the items was taken, with higher numbers reflecting greater psychological autonomy support. The confirmatory factor analysis indicated that construct validity of the instrument was good in the present study, $\chi^{2}=144.70, d f=34, p<0.001$, RMSEA $=0.08$, CFI $=0.95$, TLI $=0.93$. Internal reliability was acceptable $(\alpha=0.83)$.

\section{Personal Achievement Goal Orientations}

Students reported on their personal achievement goal orientations by responding to a 14 -item instrument. The items were selected from the Patterns of Adaptive Learning Scales (PALS) (Midgley et al., 2000), assessing students' purposes for engaging in academics. Students indicated how true $(1=$ not at all; $5=$ very true) each statement was of them: five statements tapping Mastery Goal Orientation (e.g., "It's important to me that I learn a lot of new concepts this year."), five tapping performance-approach goal orientation (e.g., "It's important to me that other students in my class think I am good at my class work.") and four tapping performance-avoidance goal orientation (e.g., "It's important to me that I don't look stupid in class."). Then, three variables were formed by summing up the scores of the corresponding items. The fit indices for the confirmatory factor test were acceptable, $\chi^{2}=264.12, d f=74, p<0.001$, RMSEA $=0.07$, CFI $=0.92$, $\mathrm{TLI}=0.90$. Internal reliabilities were acceptable $(\alpha=0.81)$.

\section{Maladaptive Academic Functioning}

Nineteen items were selected from the PALS (Midgley et al., 2000), tapping into students' academic-related beliefs, strategies, and behaviors. Students indicated how true $(1=$ not at all; $5=$ very true) each statement was of them: six tapping academic self-handicapping strategies (e.g., "Some students purposely don't try hard in class. Then if they don't do well, they can say it is because they didn't try."), five tapping avoiding novelty (e.g., "I would prefer to do class work that is familiar to me, rather than work I would have to learn how to do."), three tapping cheating behaviors (e.g., "I sometimes copy answers from other students during tests."), and five tapping disruptive behaviors (e.g., "I sometimes annoy my teacher during class."). The mean of these items was taken, with higher numbers indicating greater maladaptive academic functioning. The model of configurable factor analysis fit the data acceptable, $\chi^{2}=411.77, d f=146, p<0.001$, RMSEA $=0.06, \mathrm{CFI}=0.91, \mathrm{TLI}=0.89$. Internal reliability was acceptable $(\alpha=0.86)$.

\section{Data Analysis}

In general, the statistical analyses in the present study consisted of two steps. In the preliminary analysis, we calculated the means, SDs, and inter-correlations among the study variables. In the second step, multiple mediator analyses were conducted in which three mediators (mastery, performance-approach, and PAVs) were entered simultaneously to test the mediating role of achievement goal orientations on the relation between parental psychology control, autonomy support, and students' maladaptive academic functioning using the PROCESS syntax in SPSS (Hayes et al., 2011). The mediation method is based on regression analysis. It provided the direct effect, total effect, and indirect effects of an independent variable on a dependent variable with 95\% bias-corrected confidence intervals based on 5,000 bootstrapped samples. If the confidence intervals include 0 , the tested mediation effect would be non-significant (Preacher and Hayes, 2008). The indirect effect further calculates contrast between mediating variables in a multiple mediation model, allowing us to examine differences in the extent to which one variable mediates the relation between parenting and academic functioning as compared to other mediators included in the model.

\section{RESULTS}

\section{Preliminary Analysis}

A multivariate analysis of variance was conducted to examine the overall effects of sex on these variables. A significant effect of sex, Wilks' $\lambda=0.97, F(6,838)=4.69, p<0.001, \eta^{2}=0.03$, was found. Follow-up univariate analyses revealed that boys had higher scores on parental psychological control and maladaptive 
academic functioning $\left(\eta^{2}=0.00-0.02\right)$ than girls did. There were no significant gender differences on students' perceived parental autonomy support and their three achievement goal orientations. Means and SDs of the variables for boys and girls are presented in Table 1.

Table 2 presents the correlation matrix. The results showed that parental psychological control was related significantly positively to students' PAP $(r=0.27, p<0.01)$, PAV $(r=0.22$, $p<0.01)$, students' maladaptive academic functioning $(r=0.29$, $p<0.01)$, and not significantly to MAS $(r=-0.01, p>0.05)$. Parental autonomy support was related significantly positively to students' MAS ( $r=0.16, p<0.01)$, negatively to maladaptive academic functioning $(r=-0.13, p<0.01)$, and not significantly to students' performance-approach and PAVs $(r=0.00, r=0.02$, ps $>0.05)$. The bivariate correlation between maladaptive academic functioning and MAS $(r=-0.13, p<0.01)$ was weak negative; while the correlations between maladaptive academic functioning and PAP $(r=0.47, p<0.01)$ and PAV $(r=0.39$, $p<0.01)$ was moderate positive.

\section{Mediating Effect of Students' Achievement Goal Orientations The Coefficient for Each Path}

We hypothesized that the relation between perceived parenting and students' maladaptive academic functioning would be mediated by students' achievement goal orientations. Three regression analyses allowed us to examine mediation: (1) a regression analysis predicting effects of parenting on students' maladaptive academic functioning, (2) a regression analysis predicting students' achievement goal orientations from parenting, and (3) a regression analysis that included parenting and students' achievement goal orientations as predictors of students' maladaptive academic functioning. Students' sex was controlled as a covariate

TABLE 1 | Means and SDs of variables for boys and girls.

\begin{tabular}{lccc}
\hline Variables & Boys (447) & Girls (399) & $\boldsymbol{F}$ \\
\hline Psychological control & $2.66(0.94)$ & $2.39(0.89)$ & $19.06^{\star \star \star}$ \\
Autonomy support & $3.67(0.72)$ & $3.74(0.69)$ & 2.55 \\
Mastery goal orientation & $3.99(0.70)$ & $4.06(0.68)$ & 2.23 \\
Performance-approach goal orientation & $2.17(0.78)$ & $2.13(0.78)$ & 0.54 \\
Performance-avoidance goal orientation & $2.74(0.86)$ & $2.68(0.83)$ & 1.09 \\
Maladaptive academic functioning & $2.14(0.58)$ & $2.01(0.49)$ & $11.54^{\star \star}$ \\
\hline
\end{tabular}

SDs are in parentheses.

${ }^{* *} p<0.01,{ }^{* * *} p<0.001$. when testing the relation between parenting and maladaptive academic functioning (please see Figure 1).

First, parental psychological control and autonomy support were significant predictors of maladaptive academic functioning $\left(c_{1}=0.15, \mathrm{SE}_{c_{1}}=0.02, p<0.001 ; c_{2}=-0.07, \mathrm{SE}_{c_{2}}=0.03\right.$, $p<0.01$, respectively). Second, parental psychological control was a significant predictor of students' $\operatorname{PAP}\left(a_{2}=0.23, \mathrm{SE}_{a_{2}}=0.03\right.$, $p<0.001)$ and PAV $\left(a_{3}=0.21, \mathrm{SE}_{a_{3}}=0.03, p<0.001\right)$, but not MAS $\left(a_{1}=0.02, \mathrm{SE}_{a_{1}}=0.03, p>0.05\right)$. Parental autonomy support was only a significant predictor of students' MAS $\left(a_{4}=0.16\right.$, $\left.\mathrm{SE}_{a_{1}}=0.03, p<0.001\right)$, not $\mathrm{PAP}\left(a_{5}=0.05, \mathrm{SE}_{a_{1}}=0.04, p>0.05\right)$ and PAV $\left(a_{6}=0.06, \mathrm{SE}_{a_{1}}=0.04, p>0.05\right)$. Third, all three achievement goal orientations predicted maladaptive academic functioning $\left(b_{1}=-0.15, \mathrm{SE}_{b_{1}}=0.02, p<0.001 ; b_{2}=0.24, \mathrm{SE}_{b_{2}}=0.03\right.$, $\left.p<0.001 ; b_{3}=0.10, \mathrm{SE}_{b_{3}}=0.03, p<0.01\right)$. Moreover, parental psychological control and autonomy support still predicted maladaptive academic functioning $\left(c_{1}^{\prime}=0.08, \mathrm{SE}_{c_{1}}=0.02, p<0.001\right.$; $\left.c_{2}^{\prime}=-0.06, \mathrm{SE}_{c_{2}}=0.02, p<0.01\right)$. The results are presented in Figure 2.

\section{Indirect Effect of Parenting on Students' Maladaptive Academic Functioning}

The product of the path coefficient $(\mathrm{ab})$ for the indirect path from parenting to students' maladaptive academic functioning via students' achievement goal orientations was examined and is presented in Table 3. The specific indirect effects of psychological control on maladaptive academic functioning through approachand avoidance-performance goal orientations were both significantly different from 0 , while not significantly different through MAS. The specific indirect effect of parental autonomy support on maladaptive academic functioning through MAS was significantly different from 0 , but not significantly different through PAP and PAV.

\section{Comparison of Indirect Effects}

We further tested whether any two indirect effects of parenting variables on maladaptive academic functioning via three achievement goal orientations differed significantly. The contrasts of indirect effects from parenting on students' maladaptive academic functioning through achievement goal orientations are presented in Table 4. The two indirect effects cannot be distinguished regarding magnitude if 0 is included in the interval (Preacher and Hayes, 2008). The results indicated that the specific indirect effect of psychological control on maladaptive academic functioning through MAS was significantly different with that through PAP and PAV. The two indirect effects

TABLE 2 | Interrelations among variables.

\begin{tabular}{|c|c|c|c|c|c|c|}
\hline & 1 & 2 & 3 & 4 & 5 & 6 \\
\hline \multicolumn{7}{|l|}{1 Psychological control } \\
\hline 2 Autonomy support & $-0.14^{\star \star}$ & & & & & \\
\hline 3 Mastery goal orientation & -0.01 & $0.16^{\star \star}$ & & & & \\
\hline 4 Performance-approach goal orientation & $0.27^{\star \star}$ & 0.00 & $0.14^{\star \star}$ & & & \\
\hline 5 Performance-avoidance goal orientation & $0.22^{\star \star}$ & 0.02 & $0.22^{\star \star}$ & $0.71^{\star \star}$ & & \\
\hline 6 Maladaptive academic functioning & $0.29^{\star \star}$ & $-0.13^{\star \star}$ & $-0.13^{\star \star}$ & $0.47^{\star *}$ & $0.39^{\star \star}$ & \\
\hline
\end{tabular}

${ }^{* *} p<0.01$. 


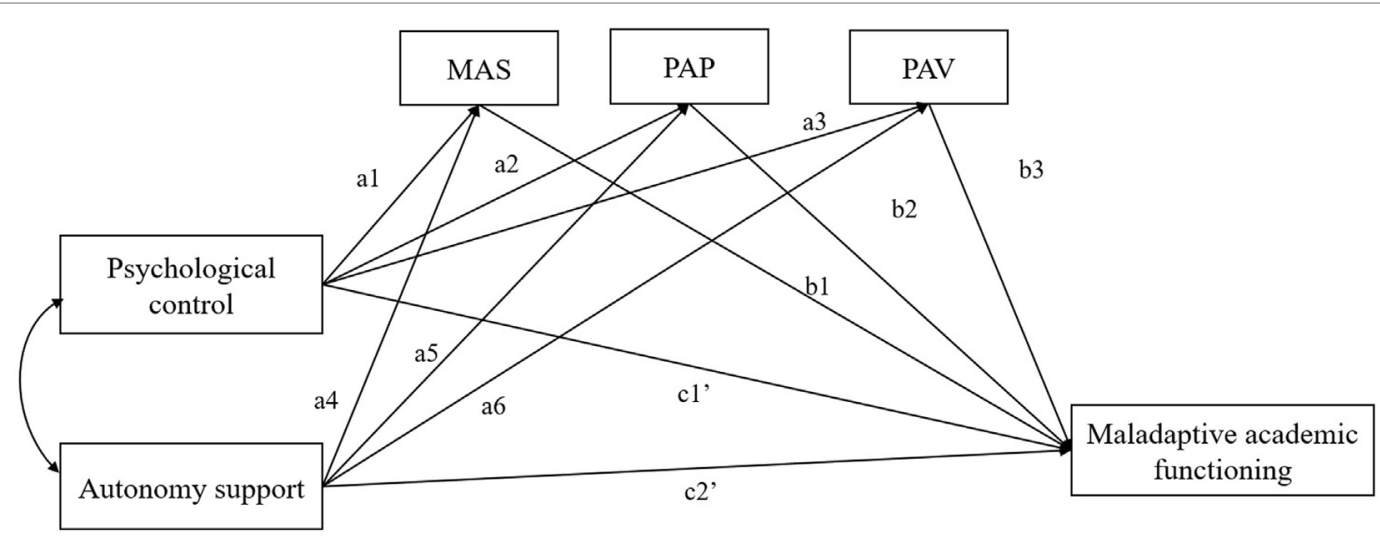

FIGURE 1 | Multiple mediation model depicting direct and indirect effects of parental psychological control and autonomy support on students' maladaptive academic functioning tested in the present study, controlling for gender. Abbreviations: MAS, mastery goal orientation; PAP, performance-approach goal orientation; PAV, performance-avoidance goal orientation.

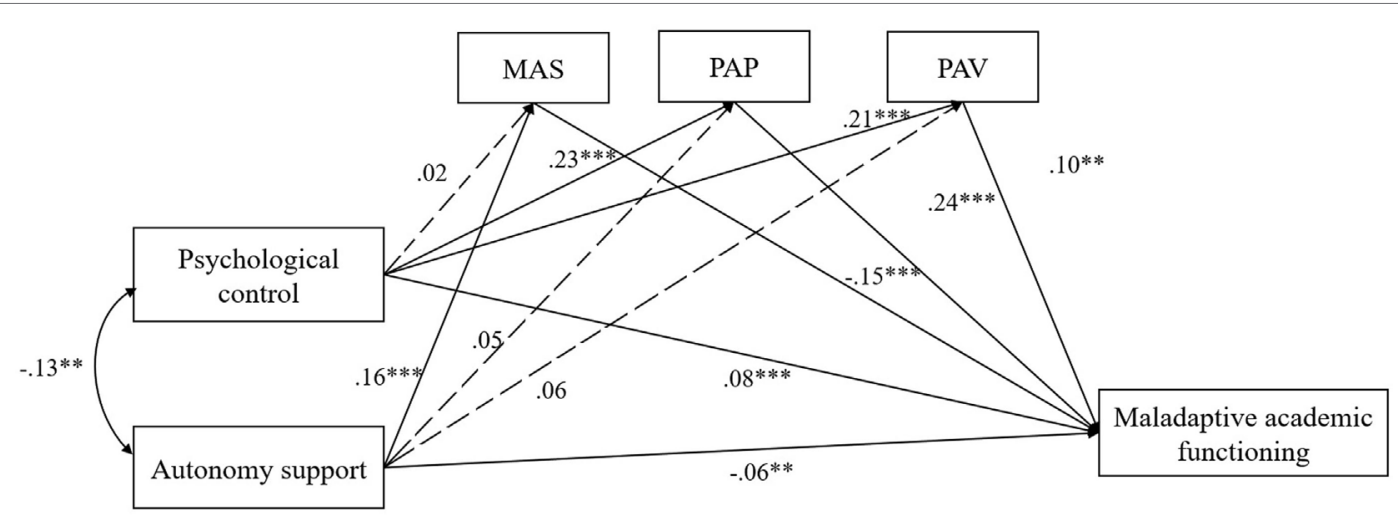

FIGURE 2 | Students' achievement goal orientations as mediators of the relation between parenting and maladaptive academic functioning. Abbreviations: MAS, mastery goal orientation; PAP, performance-approach goal orientation; PAV, performance-avoidance goal orientation. Students' gender was controlled as a covariate. Values represent standardized regression coefficients. ${ }^{\star \star} p<0.01,{ }^{\star \star \star} p<0.001$.

TABLE 3 | Indirect effect of parenting on students' maladaptive academic functioning through achievement goal orientations.

\begin{tabular}{|c|c|c|c|c|}
\hline & \multirow{2}{*}{\multicolumn{2}{|c|}{$\begin{array}{l}\text { Product of } \\
\text { coefficients }\end{array}$}} & \multirow{2}{*}{\multicolumn{2}{|c|}{$\begin{array}{c}\text { Bootstrapping } \\
\text { BC } 95 \% \mathrm{CI}\end{array}$}} \\
\hline & & & & \\
\hline & Point estimate & SE & Lower & Upper \\
\hline \multicolumn{5}{|c|}{ Psychological control } \\
\hline MAS & -0.00 & 0.01 & -0.01 & 0.01 \\
\hline PAP & 0.06 & 0.01 & 0.03 & 0.09 \\
\hline PAV & 0.02 & 0.01 & 0.01 & 0.03 \\
\hline \multicolumn{5}{|c|}{ Autonomy support } \\
\hline MAS & -0.02 & 0.01 & -0.04 & -0.01 \\
\hline PAP & 0.01 & 0.01 & -0.01 & 0.03 \\
\hline PAV & 0.01 & 0.00 & -0.01 & 0.02 \\
\hline
\end{tabular}

$B C$, bias corrected; 5,000 bootstrap samples; MAS, mastery goal orientation; PAP, performance-approach goal orientation; PAV, performance-avoidance goal orientation.

through PAP and PV can be also distinguished between each other. Moreover, the specific indirect effect of autonomy support on maladaptive academic functioning through MAS was
TABLE 4 | Contrasts of indirect effects from parenting on students' maladaptive academic functioning through achievement goal orientations.

\begin{tabular}{|c|c|c|c|c|}
\hline & \multirow{2}{*}{\multicolumn{2}{|c|}{$\begin{array}{l}\text { Product of } \\
\text { coefficients }\end{array}$}} & \multirow{2}{*}{\multicolumn{2}{|c|}{$\begin{array}{c}\text { Bootstrapping } \\
\text { BC } 95 \% \mathrm{Cl}\end{array}$}} \\
\hline & & & & \\
\hline & Point estimate & SE & Lower & Upper \\
\hline \multicolumn{5}{|c|}{ Psychological control } \\
\hline MAS vs. PAP & -0.06 & 0.02 & -0.09 & -0.03 \\
\hline MAS vs. PAV & -0.02 & 0.01 & -0.04 & -0.01 \\
\hline PAP vs. PAV & 0.03 & 0.01 & 0.01 & 0.07 \\
\hline \multicolumn{5}{|c|}{ Autonomy support } \\
\hline MAS vs. PAP & -0.04 & 0.01 & -0.07 & -0.01 \\
\hline MAS vs. PAV & -0.03 & 0.01 & -0.05 & -0.01 \\
\hline PAP vs. PAV & 0.01 & 0.01 & -0.01 & 0.02 \\
\hline
\end{tabular}

BC, bias corrected; 5,000 bootstrap samples; MAS, mastery goal orientation; PAP, performance-approach goal orientation; PAV, performance-avoidance goal orientation.

significantly different from that through PAP and PAV. However, the two indirect effects of PAP and PAV cannot be distinguished from each other. Therefore, parental psychological control leads 
to students' maladaptive academic functioning mainly through students' PAP and PAV, while parental autonomy support leads to maladaptive academic functioning only through MAS.

\section{DISCUSSION}

The present study examined adolescents' perceived parental psychological control and autonomy support and their relations with academic functioning through achievement goal orientations in Chinese adolescents. This research first systematically examined the psychometric properties of used measures with a Chinese population and then tested the mean level scores and the interrelations of these measured constructs. Moreover, a mediating effect model was tested to explore the underlying process of students' maladaptive academic functioning affected by parental psychological control and autonomy support via students' achievement goal orientations.

\section{Gender Differences}

In our study, boys perceived significantly higher parental psychological control and tended to perceive lower parental autonomy support than girls did, although not at a significant level. There may be two main reasons for this finding. First, boys may manifest more disruptive behaviors, such as excessive agitation, aggressiveness, and noncompliance than girls (Duchesne and Larose, 2007), which may make boys receive more psychological control from parents. Second, it is possible that adolescent boys expect more autonomy and self-directed than girls, and thus be more sensitive to parental psychological control and autonomy support than girls do (Werner et al., 2016).

In addition, it is worth noting that there were no significant gender differences on students' achievement goal orientations. The result of MAS is consistent with Western findings (Chouinard and Roy, 2008); however, the results of performance-approach and PAV are inconsistent with previous findings. This inconsistency may due to the emphasis on academic success and social comparison in China, along with the one-child policy. In China, academic success has great pragmatic value, such as being admitted into noteworthy universities and bringing honor to families (Ho and Hau, 2008). Chinese parents pay much attention to their children's academics. High academic achievement is viewed as a responsibility toward one's family (Chao and Tseng, 2002). Moreover, most of the participants in this study were the only child in their families. Therefore, families pin all their hopes on their child. Both Chinese girls and boys must achieve by outperforming others and avoiding looking incompetent. These explain the lack of sex differences in China as compared to the West, where boys have higher performance goal orientation than girls do (e.g., Shim et al., 2008).

\section{The Relations between Parental Psychological Control, Autonomy Support, and Adolescents' Academic Functioning}

Previous studies concerning the relations between parenting and child outcomes have been conducted mainly in Western cultures. Many researchers asserted that autonomy is not highly valued in the East; therefore, parental psychological control and autonomy support might not have the same effects on children's academic functioning as found in Western samples (Chao and Tseng, 2002). However, in our study, the results in the correlation matrix revealed that students' maladaptive academic functioning had a positive association with parental psychological control $(r=0.29, p<0.01)$ and a negative association with autonomy support $(r=-0.13, p<0.01)$. Moreover, the results of mediating analyses further showed that the total effect of parental psychological control on students' maladaptive academic functioning was positive (estimate value $=0.15$, BC 95\% CI $[0.11,0.19])$, and that of parental autonomy support was negative (estimate value $=-0.07$, BC 95\% CI $[-0.12,-0.02])$. The current findings were not in line with one recent study with Chinese adolescents (Lu et al., 2017), which indicated that the effect of maternal psychological control on academic performance should be non-significant for adolescents aged about 15 . The inconsistent findings may be related to the methodological difference in the measurement of psychological control. In our study, we tested parental psychological control without differentiating the role of mothers vs. fathers using items from Wang et al. (2007). Whereas, their study only focused on maternal psychological control using the Psychological Control Scale-Youth Self-Report (Barber, 1996). Another possible reason for this inconsistent may be due to the sample characteristics. Relatively speaking, compared with students in general high schools, selective high-school students suffer more fierce competition among students and have heavier academic pressure and expectations from their schools, teachers, and parents. With this schema, selective high-school adolescents may perceive their parents to be more psychological control than general high-school adolescents, and this may result in the inconsistency with findings of Lu et al. (2017). Anyway, findings in the present study seem to support the universalistic view that the positive relevance of autonomy support and the negative relevance of psychological control to students' maladaptive academic functioning apply to the Chinese culture, at least for selective high-school adolescents.

Moreover, in our study, both parental psychological control and autonomy support are slightly associated with adolescents' maladaptive academic functioning. The small magnitude association may be situated within cultural context (Lu et al., 2017). In Collectivist cultures, people are instilled to value interpersonal relatedness and harmony over autonomy which is prioritized and encouraged in individualist cultures (Ho, 1986). Thus, adolescents growing up in collectivist cultures, such as China, may be affected comparatively smaller by parental psychological control and autonomy support relative to individualist counterparts (Wang et al., 2007).

\section{Mediating Effects of Students' Achievement Goal Orientations}

In the study, the mediating role of students' achievement goal orientation for the relation between parental psychological control, autonomy support, and maladaptive functioning was supported. We found that, parental psychological control led to 
students' maladaptive academic functioning, mainly through students' performance-approach and PAVs rather than MAS; while parental autonomy support reduced students' maladaptive academic functioning, mainly through their MAS rather than these two types of performance goal orientations.

These findings suggest that parental psychological control and autonomy support have distinct influencing paths on adolescents' maladaptive academic functioning through achievement goal orientations. Previous studies indicated that when parents use intrusive parenting tactics to urge children to study, children may feel social-evaluative pressure and must achieve the expectations set by their parents. For instance, Roth et al. (2009) found that psychological control positively predicted children's feelings of internal compulsion to study, which in turn positively predicted standard-focused rather than interest-focused studying. Standard-focused adolescents might be more likely fear of failure in academics, which leads them to preferring PAV as well as PAP than MAS. In contrast, when parents encourage children to participate in decision-making and opinion exchanges rather than coerce children to attain ambitious standards, children are more likely to focus on learning and mastering a task rather than winning and proving their ability (Gonzalez et al., 2002). Children have their internal criteria for success or failure and have an intrinsic motivation toward learning (Ginsburg and Bronstein, 1993). From this study, we can know parenting perceived as psychological control was related to a motivational orientation where students are concerned with proving their ability and not appearing as incompetent, which enhance students' maladaptive academic functioning; while parenting perceived as autonomy support was related to students' concern of improving their ability and attaining personal mastery, which reduce students' maladaptive academic functioning.

In addition, it is worth noting that, after putting students' achievement goal orientations as mediators into the model, the direct effect of parental psychological control decreased substantially (from $c_{1}=0.15$ to $c_{1}^{\prime}=0.08$ ); while the effect of autonomy support on students' maladaptive academic functioning only changed slightly (from $c_{2}=-0.07$ to $c_{2}^{\prime}=-0.06$ ). It seems that, just comparing their absolute value and direction of the direct effects, parental autonomy support has a more direct ameliorative effect on students' maladaptive academic functioning. These results, to a certain extent, imply that parents should use more autonomy support instead of psychological control with adolescents.

\section{Contributions and Implications}

The study offered a closer look at psychological controlling and autonomy-supportive parenting in Chinese culture, responding the appeal of examining parenting within and across different countries and cultures. The findings of the study also added to a deeper understanding of how Chinese high-school students' adoption of achievement motivations vary with parental psychological control and autonomy support, and then to affect students' maladaptive academic functioning. It suggests that the harmful nature of parental psychological control and the benefit of parental autonomy support through achievement goal orientations should be concerned and addressed in Chinese parental education.

\section{Limitations and Future Directions}

The study had two main limitations. First, all data were collected from a single source, which may affect the objectivity of the conclusions. Future studies should adopt multiple sources of assessment such as including parent-reported or observed data to avoid shared method variance. Second, we did not examine the relations between parenting and students' adaptive academic functioning; therefore, we cannot compare the findings of the effects of parenting on students' maladaptive academic functioning with that on adaptive academic functioning. It is possible that the negative influence of parental psychological control exacerbates students' maladaptive academic functioning, while the positive contribution of parental autonomy support facilitates students' adaptive academic functioning. Future research should simultaneously explore the relations between parenting and students' adaptive and maladaptive academic functioning.

\section{CONCLUSION}

This novel research helps elucidate Chinese adolescents' perceived parental psychological control and autonomy support and the mediating effects of achievement goal orientations on the relations between parenting and maladaptive academic functioning. Distinct effects of two types of parenting on adolescents' maladaptive academic functioning through achievement goal orientations were revealed. Specifically, parental psychological control exacerbated students' maladaptive academic functioning, mainly through students' performance-approach and PAVs; however, parental autonomy support reduced students' maladaptive academic functioning, mainly through MAS.

\section{ETHICS STATEMENT}

This study was carried out in accordance with the recommendations of the Research Ethics Committee of East China Normal University. All research participants were not adult. Participants and their parents/legal guardians provided written informed consent in accordance with the Declaration of Helsinki. The protocol was approved by the Research Ethics Committee of East China Normal University.

\section{AUTHOR CONTRIBUTIONS}

The execution of this paper was a combined effort on behalf of all authors. CD and DD conceptualized and designed the study. The data collection was conducted by CD and ML. XX performed the statistical analyses and drafted the initial manuscript before DD, ML, and CD contributed to the writing of the manuscript. All authors approved the final version of the paper for submission.

\section{FUNDING}

This study was supported by a grant from the National Natural Science Foundation of China (grant no. 71373081) and Graduate School of East China Normal University (40600-10202$511232 / 005 / 001)$. The funding agent had no role in the study design; collection, analysis, and interpretation of the data; or writing of the manuscript. 


\section{REFERENCES}

Ames, C. (1984). Achievement attributions and self-instructions under competitive and individualistic goal structures. J. Educ. Psychol. 76, 478-487. doi:10.1037/0022-0663.76.3.478

Annear, K. D., and Yates, G. C. R. (2010). Restrictive and supportive parenting: effects on children's school affect and emotional responses. Aust. Educ. Res. 37, 63-82. doi:10.1007/bf03216914

Aunola, K., and Nurmi, J. E. (2004). Maternal affection moderates the impact of psychological control on a child's mathematical performance. Dev. Psychol. 40, 965-978. doi:10.1037/0012-1649.40.6.965

Barber, B. K. (1996). Parental psychological control: revisiting a neglected construct. Child Dev. 67, 3296-3319. doi:10.1111/j.1467-8624.1996. tb01915.x

Barber, B. K., and Harmon, E. L. (2002). "Violating the self: parental psychological control of children and adolescents," in Instructive Parenting: How Psychological Control Affects Children and Adolescents, ed. B. K. Barber (Washington, DC: American Psychological Association), 15-52.

Brislin, R. W. (1980). "Translation and content analysis of oral and written materials," in Handbook of Cross-Cultural Psychology: Vol. 2, Methodology, eds H. C. Triandis and J. W. Berry (Boston, MA: Allyn and Bacon), 389-444.

Chao, R., and Tseng, V. (2002). "Parenting of Asians," in Handbook of Parenting, ed. M. H. Bornstein (Mahwah, NJ: Lawrence Erlbaum Association Publishers), 59-93.

Chouinard, R., and Roy, N. (2008). Changes in high-school students' competence beliefs, utility value and achievement goals in mathematics. Br. J. Educ. Psychol. 78, 31-50. doi:10.1348/000709907X197993

D'Ailly, H. (2003). Children's autonomy and perceived control in learning: a model of motivation and achievement in Taiwan. J. Educ. Psychol. 95, 84-96. doi:10.1037/0022-0663.95.1.84

De Bruyn, E. H., Deković, M., and Meijnen, G. W. (2003). Parenting, goal orientations, classroom behavior, and school success in early adolescence. J. Appl. Dev. Psychol. 24, 393-412. doi:10.1016/S0193-3973(03)00074-1

Deci, E. L., and Ryan, R. M. (1985). Intrinsic Motivation and Self-Determination in Human Behavior. New York: Plenum.

Deci, E. L., and Ryan, R. M. (2010). Self-Determination. New York: John Wiley and Sons, Inc.

Deci, E. L., Ryan, R. M., and Williams, G. C. (1996). Need satisfaction and the self-regulation of learning. Learn. Individ. Differ. 8, 165-183. doi:10.1016/ S1041-6080(96)90013-8

Diseth, $\AA$, and Kobbeltvedt, T. (2010). A mediation analysis of achievement motives, goals, learning strategies, and academic achievement. Br. J. Educ. Psychol. 80, 671-687. doi:10.1016/S1041-6080(96)90013-8

Diseth, $\AA$, and Samdal, O. (2014). Autonomy support and achievement goals as predictors of perceived school performance and life satisfaction in the transition between lower and upper secondary school. Soc. Psychol. Educ. 17, 269-291. doi:10.1007/s11218-013-9244-4

Doan, S. N., Tardif, T., Miller, A., Olson, S., Kessler, D., Felt, B., et al. (2017). Consequences of 'tiger' parenting: a cross-cultural study of maternal psychological control and children's cortisol stress response. Dev. Sci. 20, e12404. doi:10.1111/desc. 12404

Duchesne, S., and Larose, S. (2007). Adolescent parental attachment and academic motivation and performance in early adolescence. J. Appl. Soc. Psychol. 37, 1501-1521. doi:10.1111/j.1559-1816.2007.00224.x

Dweck, C. S. (1986). Motivational processes affecting learning. Am. Psychol. 41, 1040-1048. doi:10.1037/0003-066X.41.10.1040

Eccles, J. S., Midgley, C., Wigfield, A., Buchanan, C. M., Reuman, D., Flanagan, C., et al. (1993). Development during adolescence: the impact of stage-environment fit on young adolescents' experiences in schools and in families. Am. Psychol. 48, 90-101. doi:10.1037/0003-066X.48.2.90

Elliot, A. J., McGregor, H. A., and Gable, S. (1999). Achievement goals, study strategies, and exam performance: a mediational analysis. J. Educ. Psychol. 91, 549-563. doi:10.1037/0022-0663.91.3.549

Erkut, S. (2010). Developing multiple language versions of instruments for intercultural research. Child Dev. Perspect. 4, 19-24. doi:10.1111/j/1750-8606. 2009.00111x

Froiland, J. M., and Worrell, F. C. (2017). Parental autonomy support, community feeling and student expectations as contributors to later achievement among adolescents. Educ. Psychol. 37, 261-271. doi:10.1080/01443410.2016.1214687
Ginsburg, G. S., and Bronstein, P. (1993). Family factors related to children's intrinsic/extrinsic motivational orientation and academic performance. Child Dev. 64, 1461-1474. doi:10.1111/j.1467-8624.1993.tb02964.x

Gonzalez, A. R., Holbein, M. F. D., and Quilter, S. (2002). High school students' goal orientations and their relationship to perceived parenting styles. Contemp. Educ. Psychol. 27, 450-470. doi:10.1006/ceps.2001.1104

Gray, M. R., and Steinberg, L. (1999). Unpacking authoritative parenting: reassessing a multidimensional construct. J. Marriage Fam. 61, 574-587. doi:10.2307/ 353561

Grolnick, W. S., Ryan, R. M., and Deci, E. L. (1991). Inner resources for school achievement: motivational mediators of children's perceptions of their parents. J. Educ. Psychol. 83, 508-517. doi:10.1037/0022-0663.83.4.508

Grouzet, F. M., Vallerand, R. J., Thill, E. E., and Provencher, P. J. (2004). From environmental factors to outcomes: a test of an integrated motivational sequence. Motiv. Emot. 28, 331-346. doi:10.1007/s11031-004-2387-z

Hagger, M. S., Sultan, S., Hardcastle, S. J., and Chatzisarantis, N. L. (2015). Perceived autonomy support and autonomous motivation toward mathematics activities in educational and out-of-school contexts is related to mathematics homework behavior and attainment. Contemp. Educ. Psychol. 41, 111-123. doi:10.1016/j.cedpsych.2014.12.002

Hayes, A. F., Preacher, K. J., and Myers, T. A. (2011). "Mediation and the estimation of indirect effects in political communication research," in Sourcebook for Political Communication Research: Methods, Measures, and Analytical Techniques, Vol. 23, eds E. P. Bucy and R. L. Holbert (New York: Routledge), 434-465.

Ho, D. Y. F. (1986). "Chinese pattern of socialization: a critical review," in The Psychology of the Chinese People, ed. M. H. Bond (Oxford: Oxford University Press), 1-37.

Ho, I. T., and Hau, K. T. (2008). Academic achievement in the Chinese context: the role of goals, strategies, and effort. Int. J. Psychol. 43, 892-897. doi:10.1080/00207590701836323

Lee, J., Yu, H., and Choi, S. (2012). The influences of parental acceptance and parental control on school adjustment and academic achievement for South Korean children: the mediation role of self-regulation. Asia Pac. Educ. Rev. 13, 227-237. doi:10.1007/s12564-011-9186-5

Lin, S. (2001). The Influence of Family Connection, Regulation, and Psychological Control on Chinese Adolescent Development. Doctoral Dissertation, University of Nebraska, Lincoln, NE. Available at: http://digitalcommons.unl.edu/ dissertations/AAI3016319

Lu, M., Walsh, K., White, S., and Shield, P. (2017). Influence of perceived maternal psychological control on academic performance in Chinese adolescents: moderating roles of adolescents' age, gender, and filial piety. Marriage Fam. Rev. 54, 1-14. doi:10.1080/01494929.2017.1347548

Luo, W., Aye, K. M., Hogan, D., Kaur, B., and Chan, M. C. Y. (2013). Parenting behaviors and learning of Singapore students: the mediational role of achievement goals. Motiv. Emot. 37, 274-285. doi:10.1007/s11031-012-9303-8

McPartland, J. M., and Epstein, J. L. (1977). Open schools and achievement: extended tests of a finding of no relationship. Sociol. Educ. 50, 133-144. doi: $10.2307 / 2112375$

Midgley, C., Maehr, M. L., Hruda, L. Z., Anderman, E., Anderman, L., Freeman, K. E., et al. (2000). Manual for the patterns of adaptive learning scales. Ann Arbor 1001, 48109-41259.

Nelson, D. A., Hart, C. H., Yang, C., Olsen, J. A., and Jin, S. (2006). Aversive parenting in China: associations with child physical and relational aggression. Child Dev. 77, 554-572. doi:10.1111/j.1467-8624.2006.00890.x

Ng, F. F. Y., Pomerantz, E. M., and Deng, C. (2014). Why are Chinese mothers more controlling than American mothers? "My child is my report card". Child Dev. 85, 355-369. doi:10.1111/cdev.12102

Nicholls, J. G. (1984). Achievement motivation: conceptions of ability, subjective experience, task choice, and performance. Psychol. Rev. 91, 328-346. doi:10.1037/0033-295X.91.3.328

Pastor, D. A., Barron, K. E., Miller, B. J., and Davis, S. L. (2007). A latent profile analysis of college students' achievement goal orientation. Contemp. Educ. Psychol. 32, 8-47. doi:10.1016/j.cedpsych.2006.10.003

Pomerantz, E. M., Ng, F. F., and Wang, Q. (2008). Culture, parenting, and motivation: the case of East Asia and the United States. Adv. Motiv. Achiev. Soc. Psychol. Perspect. 15, 209-240. doi:10.1016/S0749-7423(08)15007-5

Preacher, K. J., and Hayes, A. F. (2008). Asymptotic and resampling strategies for assessing and comparing indirect effects in multiple mediator models. Behav. Res. Methods 40, 879-891. doi:10.3758/BRM.40.3.879 
Roth, G., Assor, A., Niemiec, C. P., Ryan, R. M., and Deci, E. L. (2009). The emotional and academic consequences of parental conditional regard: comparing conditional positive regard, conditional negative regard, and autonomy support as parenting practices. Dev. Psychol. 45, 1119-1142. doi:10.1037/a0015272

Ryan, R. M., and Deci, E. L. (2003). "On assimilating identities to the self: a self-determination theory perspective on internalization and integrity within cultures," in Handbook of Self and Identity, eds M. R. Leary and J. P. Tangney (New York: Guilford Press), 253-272.

Schaefer, E. S. (1965). Children's reports of parental behavior: an inventory. Child Dev. 36, 413-424. doi:10.2307/1126465

Shim, S., Ryan, A. M., and Anderson, C. J. (2008). Achievement goals and achievement during early adolescence: examining time-varying predictor and outcome variables in growth curve analysis. J. Educ. Psychol. 100, 655-671. doi:10.1037/0022-0663.100.3.655

Silk, J. S., Morris, A. S., Kanaya, T., and Steinberg, L. (2003). Psychological control and autonomy granting: opposite ends of a continuum or distinct constructs? J. Res. Adolesc. 13, 113-128. doi:10.1111/1532-7795.1301004

Soenens, B., Park, S. Y., Vansteenkiste, M., and Mouratidis, A. (2012). Perceived parental psychological control and adolescent depressive experiences: a crosscultural study with Belgian and South-Korean adolescents. J. Adolesc. 35, 261-272. doi:10.1016/j.adolescence.2011.05.001

Steinberg, L., Lamborn, S. D., Dornbusch, S. M., and Darling, N. (1992). Impact of parenting practices on adolescent achievement: authoritative parenting, school involvement, and encouragement to succeed. Child Dev. 63, 1266-1281. doi:10.1111/j.1467-8624.1992.tb01694.x

Tynkkynen, L., Vuori, J., and Salmela-Aro, K. (2012). The role of psychological control, socioeconomic status and academic achievement in parents' educational aspirations for their adolescent children. Eur. J. Dev. Psychol. 9, 695-710. doi:10.1080/17405629.2012.671581

Vansteenkiste, M., Zhou, M., Lens, W., and Soenens, B. (2005). Experiences of autonomy and control among Chinese learners: vitalizing or immobilizing? J. Educ. Psychol. 97, 468-483. doi:10.1037/0022-0663.97.3.468
Vasquez, A. C., Patall, E. A., Fong, C. J., Corrigan, A. S., and Pine, L. (2016). Parent autonomy support, academic achievement, and psychosocial functioning: a meta-analysis of research. Educ. Psychol. Rev. 28, 605-644. doi:10.1007/ s10648-015-9329-Z

Wang, Q., Chan, H. W., and Lin, L. (2012). Antecedents of Chinese parents' autonomy support and psychological control: the interplay between parents' selfdevelopment socialization goals and adolescents' school performance. J. Youth Adolesc. 41, 1442-1454. doi:10.1007/s10964-012-9760-0

Wang, Q., Pomerantz, E. M., and Chen, H. (2007). The role of parents' control in early adolescents' psychological functioning: a longitudinal investigation in the United States and China. Child Dev. 78, 1592-1610. doi:10.1111/j. 1467-8624.2007.01085.x

Werner, L. L., Van der Graaff, J., Meeus, W. H., and Branje, S. J. (2016). Depressive symptoms in adolescence: longitudinal links with maternal empathy and psychological control. J. Abnorm. Child Psychol. 44, 1121-1132. doi:10.1007/ s10802-015-0106-8

Wolters, C. A. (2004). Advancing achievement goal theory: using goal structures and goal orientations to predict students' motivation, cognition, and achievement. J. Educ. Psychol. 96, 236-250. doi:10.1037/0022-0663.96.2.236

Conflict of Interest Statement: The authors declare that the research was conducted in the absence of any commercial or financial relationships that could be construed as a potential conflict of interest.

Copyright (c) $2018 \mathrm{Xu}$, Dai, Liu and Deng. This is an open-access article distributed under the terms of the Creative Commons Attribution License (CC BY). The use, distribution or reproduction in other forums is permitted, provided the original author(s) or licensor are credited and that the original publication in this journal is cited, in accordance with accepted academic practice. No use, distribution or reproduction is permitted which does not comply with these terms. 